\title{
INCIDENCIA Y SEVERIDAD DE LA ANTRACNOSIS EN LÍNEAS E HÍBRIDOS DE PAPAYA (Carica papaya) ${ }^{1}$
}

\author{
Antonio Bogantes-Arias ${ }^{2}$,Eric Mora-Newcomer ${ }^{2}$
}

\begin{abstract}
RESUMEN
Incidencia y severidad de la antracnosis en líneas e híbridos de papaya (Carica papaya). El objetivo de este estudio fue determinar la incidencia y severidad de la antracnosis (Colletotrichum gloeosporioides), en frutas de papaya. El experimento se realizó en Guápiles, Limón, Costa Rica, entre los años 2010 y 2011. Se sembraron cinco líneas y cuatro híbridos bajo un diseño de bloques completos al azar con tres repeticiones. Se evaluó la incidencia y severidad de la antracnosis, así como la firmeza y los sólidos solubles (grados Brix) de la fruta. Los híbridos presentaron características intermedias a los padres para las características evaluadas. En la antracnosis de fruta, la resistencia tiende a ser dominante para el material evaluado.
\end{abstract}

Palabras clave: Colletotrichum gloeosporioides, poscosecha en frutas de papaya, resistencia a la antracnosis en papaya.

\begin{abstract}
Incidence and severity of anthracnose in breeding lines and crosses of papaya (Carica papaya). The objective of this study was to determine the incidence and severity of papaya fruit anthracnose (Colletotrichum gloeosporioides). The experiment was carried out in Guápiles, Limón, Costa Rica, between the years 2010 and 2011. Five lines and four crosses were evaluated in a randomized complete block design with three replications. Evaluated traits were incidence and severity of anthracnose, fruit firmness and total soluble solids content of the pulp. Results suggest that the crosses have intermediate characteristics to those of their parent lines for these traits. In the specific case of anthracnose, evidence also suggests that the resistance to this disease tends to be inherited in a dominant manner.
\end{abstract}

Keywords: Colletotrichum gloeosporioides, postharvest life of papaya fruit, anthracnose resistance in papaya.

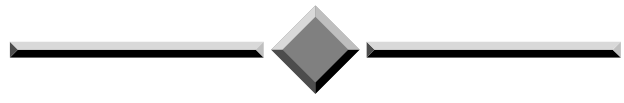

\footnotetext{
1 Recibido: 19 de octubre, 2011. Aceptado: 28 de octubre, 2013. Trabajo realizado como parte del proyecto de Mejoramiento de Variedades Vegetales. Convenio Universidad de Costa Rica (UCR) - Instituto Nacional de Innovación y Transferencia en Tecnología Agropecuaria (INTA).

2 Programa de Frutales, Estación Experimental Los Diamantes, Instituto Nacional de Innovación y Transferencia en Tecnología Agropecuaria. Costa Rica. Tel. (506) 271044 08. abogantes@inta.go.cr

3 Programa de Frutales, Estación Experimental Agrícola Fabio Baudrit, Universidad de Costa Rica. Costa Rica. Tel. (506) 25 11 77 69. Apdo. postal 183-4050 Alajuela, Costa Rica.emoran@racsa.co.cr
} 


\section{INTRODUCCIÓN}

La antracnosis (Colletotrichum gloeosporioides) de la fruta es una de las enfermedades fúngicas más importantes de la papaya. Los síntomas son diversos y pueden manifestarse como lesiones redondas y hundidas o bien como manchas más superficiales de color café, que pueden abarcar grandes extensiones en el fruto (Durán y Mora 1987).

Las esporas del hongo comúnmente atacan la fruta en estado verde, pero se pueden mantener latentes durante meses hasta que se inicia la maduración, momento en el cual se presentan los cambios físicos y químicos necesarios para el desarrollo de las lesiones (Dickman y Álvarez 1983, Umaña 1998, Tatagiba et al. 2002). Los peciolos senescentes de las hojas son fuente importante de inóculo para esta enfermedad (Castro et al. 1996) y la remoción de los mismos puede ser una práctica efectiva de control (Durán et al. 2000). Las condiciones de alta precipitación y humedad relativa favorecen el desarrollo de la enfermedad (Durán et al. 1998, Tatagiba et al. 2002).

Las pérdidas causadas en papaya por este hongo pueden llegar a ser muy cuantiosas. Por ejemplo, en Brasil, uno de los principales países productores del mundo, se han reportado pérdidas de hasta un $90 \%$ bajo condiciones ambientales que favorecen el desarrollo de esta enfermedad (Tatagiba et al. 2002). En Costa Rica, Solano y Arauz (1995) reportan incidencias de antracnosis entre 40 y $90 \%$ en tratamientos testigo, en los cuales no se realizó ningún control químico. Desde el punto de vista económico, las pérdidas por esta enfermedad sobrepasan el 24\% (Demerutis 1994) y es la principal causa de rechazo para la exportación hacia el mercado canadiense $e^{4}$.

El control de la antracnosis se realiza en campo, proporcionándole al cultivo una buena condición sanitaria, con prácticas culturales y con un programa de aplicación preventiva de fungicidas protectantes como mancozeb y clorotalonil (Solano y Arauz 1995). El prochloraz es una opción para el tratamiento poscosecha en frutos que vienen inoculados del campo (Zavala et al. 2005, Santamaría et al. 2011). Una limitante en el control químico de esta enfermedad en nuestro

4 Barrientos, M. 2010. Antracnosis (Colletotrichum gloeosporiodes) en papaya para exportación. Centro Agrícola Cantonal de Guácimo. Guácimo, Pococí. Costa Rica. Comunicación personal. país es la poca cantidad de productos registrados en el cultivo, así como, la resistencia del hongo a fungicidas como tiabendazol, benomil y la carbendazina (Astúa et al 1994, Gutiérrez y Gutiérrez 2003).

La incidencia y la severidad de los síntomas en la fruta es una variable que casi siempre se evalúa en las pruebas de cultivares (Acosta et al. 2001, Mora y Bogantes 2004a) y resulta evidente que la resistencia al patógeno es una característica importante en los procesos de selección y producción de nuevas líneas e híbridos de este cultivo (Mora y Bogantes 1999-2002), pero muy pocos trabajos de investigación están relacionados con la búsqueda de resistencia o con el modo de herencia de la antracnosis en papaya. Una notable excepción es el trabajo de Vivas et al. (2011), quienes reportan la habilidad combinatoria y la contribución de algunas líneas a la resistencia de la antracnosis en la fruta.

El objetivo de este estudio fue determinar la incidencia y severidad de la antracnosis (C. gloeosporioides), los sólidos solubles y la firmeza en frutas de papaya en un grupo particular de líneas genéticas y algunos de sus híbridos.

\section{MATERIALES Y MÉTODOS}

El experimento se inició en marzo del 2010 y finalizó en febrero del 2011, en la finca de la Estación Experimental Los Diamantes, localizada en el cantón de Pococí, provincia de Limón, Costa Rica. La altura aproximada de dicha región es de $225 \mathrm{msnm}$, con una temperatura promedio de $24,6^{\circ} \mathrm{C}$ y una precipitación anual promedio de $4380 \mathrm{~mm}$.

Los tratamientos fueron líneas e híbridos producidos en el marco del proyecto conjunto entre la Universidad de Costa Rica (UCR) y el Instituto Nacional de Innovación y Transferencia de Tecnología Agropecuaria (INTA) para la mejora genética de la papaya (Cuadro 1).

Las líneas progenitoras utilizadas tienen diferentes orígenes:

C06-P2 es una línea estable, producida a través de autopolinizaciones de una selección de un material criollo.

Las líneas HC70-110, HC05-217, MH04-143 y HC03-ST4 son estables, derivadas del cruzamiento de material autóctono con introducciones de Cuba y Hawaii (Mora y Bogantes 1999-2002). 
Cuadro 1. Líneas de papaya con sus respectivos híbridos. Guápiles, Limón, Costa Rica. 2012.

\begin{tabular}{ll}
\hline & Tratamientos \\
\hline Líneas & Híbridos \\
\hline HC03-ST4 & HC03-ST4 x HC05-217 \\
HC70-110 & HC70-110 x HC05-217 \\
C06-P2 & C06-P2 x HC05-217 \\
MH04-143 & MH04-143 x HC05-217 \\
HC05-217 & \\
\hline
\end{tabular}

Los híbridos se seleccionaron con el objetivo de determinar la reacción de la fruta de los diferentes materiales ante la antracnosis. Se incluyeron algunas líneas élite del programa de mejora. La línea HC03-ST4 es considerada como resistente a la antracnosis debido a la poca incidencia y severidad en fruta durante cinco ciclos de autofecundación, la cual ha estado en ambas características por debajo del $5 \%$ en todo su proceso de selección como línea. Por el contrario, la línea HC05-217 se seleccionó por presentar una alta susceptibilidad a esta enfermedad durante cuatro periodos de autofecundación, donde mostró $100 \%$ de incidencia y más de $40 \%$ de severidad en fruta (Cuadro 1).

Los cruzamientos se hicieron en el campo de manera controlada para garantizar la pureza de los híbridos, siguiendo procedimientos básicos para evitar la contaminación genética.

Las condiciones de clima durante el experimento se resumen en el Cuadro 2 y se pueden considerar típicas para la zona.

Durante el estudio no se consideró necesario hacer una inoculación artificial del hongo a las frutas debido a que existen diferentes razas, cepas o variantes de
C. gloesporioides que infectan la fruta de la papaya (Durán et al. 1999, Casarrubias et al. 2003, Londoño et al. 2007), la inoculación de una sola raza no daría resultados representativos semejantes a lo que ocurre en las plantaciones comerciales, en las cuales no se conoce cuál es la raza predominante. Razones que justifican esta decisión:

- Las hojas y peciolos con lesiones de la enfermedad se dejaron desprender naturalmente y no se removieron del campo, sino que se acumularon en la base de cada planta como fuente comprobada de inóculo (Durán et al. 2000).

- Las condiciones ambientales durante todo el experimento (Cuadro 2) fueron consideradas como muy favorables para el desarrollo de la enfermedad (Durán et al. 1998, 1999, Tatagiba et al. 2002).

- No se utilizó ningún control químico contra el hongo.

- La fruta se dejó madurar en su totalidad en la planta para exponerla a más presión de inóculo natural.

El diseño experimental consistió en tres bloques completos al azar con nueve tratamientos en cada bloque. Estos estuvieron constituidos por parcelas de cinco plantas, y la parcela útil fueron las tres plantas sembradas en el centro a 2,5 m entre sí y 2,5 m entre surco.

La fertilización se hizo con la fórmula comercial 10-30-10 (30-60 g/planta) aplicados a la siembra, al primer y segundo mes de edad de las plantas. A partir del tercer mes se aplicó mensualmente la fórmula 18-515-6-1,2 (100 g/planta), lo cual se complementó mensualmente con aplicaciones foliares de microlementos.

Las variables evaluadas fueron:

Incidencia (\%) de antracnosis. Porcentaje de frutas con antracnosis cuando las mismas habían alcanzado la maduración, indicado por el cambio de color de la cáscara de verde a un $100 \%$ amarillo, que

Cuadro 2. Datos climáticos durante los quince meses de la evaluación de la incidencia de antracnosis en papaya. Guápiles, Limón, Costa Rica. 2011.

\begin{tabular}{lccccc}
\hline & \multicolumn{3}{c}{ Temperatura $\left({ }^{\circ} \mathbf{C}\right)$} & & \\
\cline { 2 - 4 } Promedio semanal & Máxima & Promedio & Mínima & Humedad (\%) & Precipitación (mm) \\
\hline Valor mínimo & 28,2 & 21,6 & 16,4 & 86,5 & 2,0 \\
Promedio general & 31,5 & 24,4 & 19,6 & 92,0 & 80,8 \\
Valor máximo & 33,6 & 26,5 & 22,1 & 97,0 & 249,8 \\
Acumulado & & & & & 5168 \\
\hline
\end{tabular}


corresponde al estado E7 de la escala de maduración en papaya Pococí (Salazar 2012).

Severidad (\%) de antracnosis en fruta. Se calculó haciendo una observación visual del porcentaje de síntomas en la fruta madura en plantas a partir de los diez meses de edad.

Las muestras para incidencia y severidad de antracnosis constó de 32 frutos provenientes de tres plantas por repetición, para un total de 96 frutas por tratamiento.

Para hacer una adecuada estimación de la antracnosis en comparaciones de tratamientos en papaya, Liberato et al. (2004) sugieren hacer al menos siete cosechas bisemanales. En este estudio se hicieron doce cosechas con una frecuencia semanal desde octubre del 2010 hasta enero 2011.

Sólidos solubles (grados Brix) de la pulpa y firmeza (Newton) de cáscara y pulpa. Se evaluaron en frutas totalmente maduras de plantas a partir de nueve meses de edad. La muestra para firmeza de la pulpa y grados Brix se tomó de seis frutas de tres plantas por repetición, para un total de dieciocho por tratamiento.

Los grados Brix se midieron con un refractómetro de mano graduado de 0 a $32 \%$. La firmeza se midió penetrando cáscara y pulpa, con un penetrómetro manual de 200 newton (N), con un extremo (accesorio) de punta plana y redonda de $1 \mathrm{~cm}$ de diámetro.

Los datos finales fueron sometidos a pruebas de normalidad (Shapiro-Wilk) y homogeneidad de varianzas. Los datos de porcentaje de severidad y firmeza fueron transformados a su raíz cuadrada previo al análisis de varianza y su respectiva separación de medias. También se realizaron correlaciones entre las variables severidad, grados Brix y firmeza tanto en las líneas como en los híbridos.

Todos los análisis fueron realizados utilizando el programa estadístico SAS System, versión 9 con el apoyo del respectivo manual para el análisis de experimentos agrícolas (López y López 1995).

\section{RESULTADOS Y DISCUSIÓN}

\section{Incidencia y severidad de antracnosis}

El número de frutas con antracnosis (incidencia), así como el porcentaje de daño en la fruta (severidad) fue diferente entre las líneas $(\mathrm{p}<0,0001$ y $\mathrm{p}<0,0001$, respectivamente) (Cuadros 3 y 4 ).
Cuadro 3. Incidencia de antracnosis (\%) en fruta de líneas de papaya y sus respectivos híbridos con la línea HC05-217. Guápiles, Limón. Costa Rica. 2011.

\begin{tabular}{llll}
\hline \multicolumn{4}{l}{ Tratamientos } \\
\hline Líneas & & Híbridos & \\
\hline HCO3-ST4 & 10 e & C06-P2 X HC05-217 & 54 d \\
C06-P2 & 70 bcd & HCO3-ST4 X HC05-217 & 62 d \\
MH04-143 & 86 abc & HC70-110 X HC05-217 & 68 abc \\
HC70-110 & 89 ab & MH04-143 X HC05-217 & 82 dc \\
HC05-217 & $100 \mathrm{a}$ & & \\
\hline
\end{tabular}

Tratamientos con diferente letra en una misma columna o línea presentan diferencias significativas según Prueba de Duncan al $5 \%$.

Cuadro 4. Severidad de antracnosis (\%) en fruta de líneas de papaya y sus respectivos híbridos con la línea HC05-217 ( $\mathrm{n}=16$ frutas). Guápiles, Limón, Costa Rica. 2011.

\begin{tabular}{llll}
\hline \multicolumn{4}{c}{ Tratamientos } \\
\hline Líneas & & Híbridos \\
\hline HCO3-ST4 & 1,17 d & HCO3-ST4 X HC05-217 & 7,33 c \\
C06-P2 & 6,67 c & C06-P2 X HC05-217 & $6,50 \mathrm{c}$ \\
MH04-143 & 16,50 b & HC70-110X HC05-217 & 7,83 b \\
HC70-110 & 17,33 b & MH04-143 X HC05-217 & 14,83 c \\
HC05-217 & 36,00 a & & \\
\hline
\end{tabular}

Tratamientos con diferente letra en una misma columna o línea presentan diferencias significativas según Prueba de Duncan al $5 \%$.

El $100 \%$ de los frutos de la línea HC05-217 presentaron antracnosis, con un promedio de $36 \%$ de severidad en la fruta. Esta línea se podría considerar como la más susceptible de todas las evaluadas. Otras líneas, como la MH04-143 y la HC70-110, también presentaron una alta susceptibilidad, con incidencias y severidades por encima del 80 y $16 \%$, respectivamente (Cuadros 3 y 4).

La línea HCO3-ST4 presentó solo un 10\% de incidencia y una severidad de $1,17 \%$ en la fruta (Cuadros 3 y 4). Uno de los objetivos principales del desarrollo de esta línea fue la resistencia a este patógeno. 
La línea C06-P2 mostró una alta incidencia (70\%) de antracnosis pero con una baja severidad $(6,7 \%)$ en la fruta (Cuadros 3 y 4).

Los híbridos entre las líneas MH04-143 x HC05217 y HC70-110 x HC05-217 tuvieron la mayor incidencia y severidad $(\mathrm{p}=0,007)$ de antracnosis en la fruta analizada respecto al resto.

Los resultados parecen sugerir que la incidencia presentada en la fruta de los híbridos fue menor o cercana al promedio de ambos padres, pero la separación de medias no permite establecer esta relación con toda seguridad (Cuadro 3). Sin embargo, los resultados obtenidos en cuanto a la severidad parecen indicar la existencia de un efecto de dominancia a la resistencia (Cuadro 4). En este sentido, el porcentaje de fruta afectada de los híbridos que involucraron las líneas más resistentes (HCO3-ST4 y C06-P2) con la línea altamente susceptible (HC05-217), correspondió respectivamente a 7,33 y $6,50 \%$, muy por debajo $(61 \%$ y $70 \%$, respectivamente) del daño esperado cuando se promedia la severidad en ambos progenitores. Una tendencia similar a lo anteriormente señalado se observa en los híbridos que involucran las otras dos líneas medianamente susceptibles (HC70-110 y MH04-143).

En general, parece que existió una tendencia hacia la dominancia de la resistencia. Vivas et al. (2011) encontraron una contribución de algunas líneas a la resistencia de la antracnosis en las frutas; estos autores consideran que los híbridos y líneas más resistentes aportaron un avance importante en cuanto a la disminución de pérdidas poscosecha. Solano y Arauz (1995); reportan un 90\% de incidencia, así como una severidad del $13 \%$, después de seis días de cosecha en el tratamiento testigo (sin ninguna aplicación de fungicidas), en variedades criollas, sembradas en esta misma zona. En tres de los híbridos de este trabajo, el porcentaje de severidad fue inferior al $8 \%$.

\section{Firmeza de fruta}

La firmeza de la fruta en el estado de consumo, fue diferente entre líneas y entre híbridos $(p<0,0001)$. En términos generales, la línea HC05-217 con una firmeza de $164 \mathrm{~N}$, fue entre tres y cuatro veces más firme que las demás, las cuales, variaron entre 34 y $53 \mathrm{~N}$ (Cuadro 5). Se considera que la firmeza de la línea mencionada es elevada, ya que genera dificultad para partir la fruta y extraerle la pulpa con una cuchara. La susceptibilidad
Cuadro 5. Firmeza y grados Brix en fruta de líneas de papaya y sus respectivos híbridos con la línea HC05-217. Guápiles, Limón, Costa Rica. 2011.

\begin{tabular}{lcc}
\hline Tratamiento & Firmeza $(\mathbf{N})$ & Brix \\
\hline Líneas & & \\
HC05-217 & $164 \mathrm{a}$ & $10,33 \mathrm{~b}$ \\
HCO3-ST4 & $47 \mathrm{ef}$ & $11,17 \mathrm{ab}$ \\
HC70-110 & $53 \mathrm{e}$ & $10,67 \mathrm{ab}$ \\
C06-P2 & $50 \mathrm{e}$ & $8,50 \mathrm{c}$ \\
MH04-143 & $34 \mathrm{f}$ & $11,00 \mathrm{ab}$ \\
Híbridos & & \\
HC70-110 x HC05-217 & $110 \mathrm{~b}$ & $11,00 \mathrm{ab}$ \\
C06-P2 x HC05-217 & $73 \mathrm{~cd}$ & $11,00 \mathrm{ab}$ \\
HCO3-ST4 x HC05-217 & $61 \mathrm{de}$ & $10,67 \mathrm{ab}$ \\
MH04-143 x HC05-217 & $53 \mathrm{e}$ & $11,50 \mathrm{a}$ \\
\hline
\end{tabular}

Tratamientos con diferente letra en una misma columna presentan diferencias significativas según Prueba de Duncan al 5\%.

a la magulladura de la fruta de papaya es una limitante importante para su comercialización y está relacionado con la firmeza (Khurnpoon y Siriphanich 2012). Según observaciones de los autores, una resistencia a la penetración (firmeza) de $50 \mathrm{~N}$ es aceptable para fruta madura que no requiere de mucha manipulación; sin embargo, cuando se requiera más vida de anaquel o transportar a destinos alejados, es deseable una resistencia cercana a los $80 \mathrm{~N}$ (Osuna et al. 2005). Fonseca et al. (2003) señalan que una baja firmeza de pulpa promueve menor resistencia al transporte y obliga a establecer mayores cuidados para el manejo postcosecha.

La firmeza de fruta de todos los híbridos que involucraron la línea HC05-217 fue mayor con respecto a la de sus parentales menos firmes. En casi todos los casos, el aumento en la firmeza fue significativa; esto sugiere un potencial para utilizar cultivares de fruta muy firme para mejorar esta característica en híbridos comerciales. El material MH04-143 presentó la fruta significativamente más suave del grupo. La baja firmeza de la fruta le restó totalmente el valor comercial a esta línea; sin embargo, las frutas de su híbrido con la línea extrafirme HC05-217 presentaron una firmeza significativamente mayor, por encima de los 50N mínimos aceptables (Cuadro 5). 
Se ha relacionado mayor firmeza de la fruta con un menor daño por antracnosis (Acosta et al. 2001), pero los resultados obtenidos con los materiales evaluados, mostraron un comportamiento diferente. Así por ejemplo, la línea HC05-217 de mayor firmeza, fue también la que presentó la mayor incidencia y severidad de antracnosis, en tanto que la línea HCO3-ST4, una de las de menor firmeza, presentó a la vez una baja incidencia y severidad. Sin embargo, estos resultados tampoco permiten deducir que la relación sea inversa, ya que también se observa que la línea MH04-143 resultó con la fruta menos firme de todas las evaluadas pero con un alto grado de incidencia y severidad por antracnosis.

\section{Grados Brix de la fruta}

Solamente la línea C06-P2 difirió significativamente de las demás líneas e híbridos para la característica de grados Brix de pulpa, al presentar valores inferiores para este parámetro (Cuadro 5). El cruzamiento de este material con la línea $\mathrm{HC} 05-217$, produjo un híbrido con altos grados Brix, lo cual parece indicar un efecto dominante de esta línea en los altos contenidos de azúcares. Esta tendencia ya había sido reportada por los autores en el 2004 (Mora y Bogantes 2004b).

La correlación entre la severidad del daño por antracnosis con los grados Brix en la fruta no fue significativa entre líneas $(r=-0,32, p=0,54)$ ni entre híbridos $(r=-0,70, p=0,12)$, corroborando lo señalado por Acosta et al. (2001), quienes concluyeron en sus estudios que el grado de daño por antracnosis no afectó los grados Brix en la fruta.

\section{AGRADECIMIENTOS}

Un agradecimiento al joven Luis Felipe Rojas B., técnico del proyecto y al señor Luis Aguirre trabajador de la estación Los Diamantes por su colaboración en el mantenimiento y en las evaluaciones de campo.

\section{LITERATURA CITADA}

Astúa, G; Arauz, F; Umaña, G. 1994. Sensibilidad reducida al tiabendazole en Colletotrichum gloeosporioides ailado de papaya. Agronomía Costarricense 18(1):35-39.

Acosta, M; Nieto, D; Domínguez, J; Delgadillo, F. 2001. Calidad y tolerancia en frutos de papaya (Carica papaya L.) a la inoculación del hongo Colletotrichum gloeosporioides Penz., en postcosecha. Revista Chapingo. Serie Horticultura 7(1):119-130.

Casarrubias U; González, M; Cruz, A; Cárdenas, E; Nieto, D; Guevara, R. 2003. Variabilidad genética de Colletotrichum gloeosporioides (PENZ.) aislado de frutos de papaya (Carica papaya L.) mediante el uso de marcadores moleculares RAPD. Revista Mexicana de Fitopatología 21(3):338-345.

Castro, O; Arauz, F; Wang, A. 1996. Importancia de los pecíolos de papaya como fuente de inóculo de la antracnosis del fruto de papaya. In Bertsch, F; Rivera, G; Mora, F; Navarro, JR; Badilla, W. eds. Memoria $\mathrm{X}$ Congreso Nacional Agronómico y de Recursos Naturales, III Congreso Nacional de Fitopatología, II Congreso Nacional de Suelos. San José, Costa Rica, EUNED, EUNA. p. 123.

Demeritus, C. 1994. Diagnóstico de pérdidas poscosecha de naranja y papaya. In Memoria I Taller Regional de manejo Poscocsecha de Productos de Interés para el Trópico. San José, Costa Rica, Universidad de Costa Rica. sp.

Dickman, MB; Álvarez, AM. 1983. Latent infection of papaya caused by Colletotrichum gloeosporioides. Plant Disease 67:748-750.

Durán, JA; Mora, D. 1987. Variabilidad de la sintomatología de la antracnosis (Colletotrichum sp.) en papaya durante el período poscosecha en Costa Rica. Agronomía Costarricense 11(2):155-161.

Durán, A; Ramírez, L; Mora, D. 1998. Requerimentos de humedad relativa y tiempos de incubación para la infección de conidios de Colletotrichum gloeosporioides en frutos de papaya. Agronomía Mesoamericana 9(1):81-85.

Durán, A; Mora, D; Chavarría, E. 1999. Determinación de la edad susceptible del fruto de la papaya (Carica papaya L.) a la antracnosis (Colletotrichum gloeosporioides). (PENZ.). Agronomía Mesoamericana 10(1):01-06.

Durán A; Mora, D; Ramírez, L. 2000. Los peciolos de la papaya como fuente de inóculo de la antracnosis y su eliminación como práctica de control. Agronomía Mesoamericana 11(2):07-14.

Fonseca, M; Rocha A, N; Cenci, S; Cecon, P; Bressan R. 2003. Comparación entre las papayas var. Sunrise Solo y var. Golden, durante siete estados de madurez. Revista Iberoamericana de Tecnología Postcosecha 5(2):86-91.

Gutiérrez, O; Gutiérrez, J. 2003. Evaluación de resistencia a benomil tiabendazol, y azoxystrobin para el control de 
antracnosis (Colletotrichum gloeosporioides) en frutos de guayaba (Psidium guajava L.) en postcosecha. Revista Mexicana de Fitopatología 2(2):228-232.

Khurnpoon, L; Siriphanich, J. 2012. Change in fruit quality and cell wall polysaccharides in bruised papaya cultivars "Khak Dum" and "Holland". Acta Hort. 945:381-389.

Liberato, J; Cruz, C; Tatagiba, J; Zambolim, L. 2004. Minimum number of assessment times to compare chemical control treatments for papaya fruit anthracnose. Fitopatologia Brasileira 29(6):659-662.

Londoño, G; Lavelet,L; Galindo, M; Afanador,L. 2007. Uso de métodos multivariantes para la agrupación de aislamientos de Colletotrichum spp. con base en características morfológicas y culturales. Revista Facultad Nacional de Agronomía- Medellín 60(1):3671-3690 (en línea). Consultado 20 feb. 2012. Disponible en http://www.scielo.org.co/scielo.php?script=sci_arttext\&pid=S030428472007000100004\&lng=en\&nrm=iso

López, G; López, J. 1995. Introducción al micro SAS: Aplicación al análisis de experimentos agrícolas. Unidad de Información y Bioestadística. CATIE, Turrialba. Costa Rica. 119 p.

Mora, E; Bogantes, A. 1999-2002. Evaluación del potencial comercial de líneas S2 de papaya (Carica papaya L.) en la Región Atlántica de Costa Rica. Revista de Agricultura Tropical 32:73-80.

Mora, E; Bogantes, A. 2004a. Evaluación de híbridos de papaya (Carica papaya) en Pococí, Limón, Costa Rica. Agronomía Mesoamericana 15(1):39-44.

Mora, E; Bogantes, A. 2004b. Herencia de la concentración de los sólidos solubles entre líneas parentales de papaya (Carica papaya) y sus híbridos. Agronomía Mesoamericana 15(1):81-84.

Osuna, JA; Beltrán, JA; Pérez, MH. 2005. Mejoramiento de vida de anaquel y calidad de papaya Maradol con 1-Metilciclopropeno (1-MCP). Revista Chapingo, serie Horticultura 11(1):7-12.

Salazar, D. 2012. Establecimiento de la escala de madurez y potencial de vida útil durante el almacenamiento de papaya (Carica papaya L.) híbrido 'Pococí'. Tesis Ing. Agr. San José, CR. Escuela de Agronomía, Facultad de Ciencias Agroalimentarias, Universidad de Costa Rica. 91 p.

Santamaría, F; Díaz, R; Gutiérrez, O; Santamaría J; Larqué, A. 2011. Control de dos especies de Colletotrichum causantes de antracnosis en frutos de papaya Maradol. Revista Mexicana de Ciencias Agrícolas 2(5):631643.

Solano, V; Arauz, LF. 1995. Combate de antracnosis en frutos de papaya mediante aplicaciones de fungicidas en el campo en la zona Atlántica de Costa Rica. Agronomía Costarricense 19(2):25-30.

Tatagiba, J; Liberato, J; Zambolim, L; Ventura, J; Costa, H. 2002. Control y condiciones climáticas favorables a antracnosis de papaya. Fitopatología Brasileira 27(2):186-192.

Umaña, G. 1998. Elementos de patología postcosecha. In Báez, R. ed. Manejo postcosecha de frutales y verduras en Iberoamérica. Programa Iberoamericano de Ciencia y Tecnología para el desarrollo. Hermosillo, Sonora, México. p. 63-73.

Vivas, M; Silvaldo, F; Pessancha, C; Gonzaga, M. 2011. Testers for combining ability and selection of papaya hybrids resistant to fungal diseases. Crop Breeding and Applied Biotechnology 11:36-42.

Zavala, M; Suárez, J; Alejo, J; Ruiz, E; Gutiérrez, O; Vázquez, M; Méndez, R. 2005. Control poscosecha de antracnosis en papaya y sensibilidad de Colletotrichum gloeosporioides a fungicidas organosintéticos. Revista Chapingo, serie Horticultura 11(2):251-255. 
